\title{
Characterisation of alternative expression vectors for recombinant Bacillus Calmette-Guérin as live bacterial delivery systems
}

\author{
Larissa V Nascimento ${ }^{1,2}$, Carina C Santos ${ }^{3}$, Luciana CC Leite 1 , Ivan P Nascimento ${ }^{1 /+}$ \\ ${ }^{1}$ Instituto Butantan, Laboratório Especial de Desenvolvimento de Vacinas, São Paulo, SP, Brasil \\ ${ }^{2}$ Universidade de São Paulo, Programa de Pós-Graduação Interunidades em Biotecnologia, São Paulo, SP, Brasil \\ ${ }^{3}$ Universidade Federal da Bahia, Faculdade de Farmácia, Departamento de Análises Clínicas e Toxicológicas, Salvador, BA, Brasil
}

BACKGROUND Bacillus Calmette-Guérin (BCG) is considered a promising live bacterial delivery system. However, several proposals for $\mathrm{rBCG}$ vaccines have not progressed, mainly due to the limitations of the available expression systems.

OBJECTIVES To obtain a set of mycobacterial vectors using a range of promoters with different strengths based on a standard backbone, previously shown to be stable.

METHODS Mycobacterial expression vectors based on the pLA71 vector as backbone, were obtained inserting different promoters $\left(\mathrm{P}_{\mathrm{AN}}, \mathrm{P}_{\alpha \mathrm{Ag}}, \mathrm{P}_{\mathrm{Hsp} 60}, \mathrm{P}_{\mathrm{BlaF}}\right.$ and $\left.\mathrm{P}_{\mathrm{L} 5}\right)$ and the green fluorescence protein (GFP) as reporter gene, to evaluate features such as their relative strengths, and the in vitro (inside macrophages) and in vivo stability.

FINDINGS The relative fluorescence observed with the different vectors showed increasing strength of the promoters: $\mathrm{P}_{\mathrm{AN}}$ was the weakest in both Mycobacterium smegmatis and BCG and $\mathrm{P}_{\mathrm{BlaF}^{*}}$ was higher than $\mathrm{P}_{\mathrm{Hsp} 60}$ in BCG. The relative fluorescence observed in a macrophage cell line showed that $\mathrm{P}_{\mathrm{BlaF} *}$ and $\mathrm{P}_{\mathrm{Hsp} 60}$ were comparable. It was not possible to obtain strains transformed with the extrachromosomal expression vector containing the $\mathrm{P}_{\mathrm{L} 5}$ in either species.

MAIN CONCLUSION We have obtained a set of potentially stable mycobacterial vectors with a arrange of expression levels, to be used in the development of $\mathrm{rBCG}$ vaccines.

Key words: recombinant BCG - expression vectors - promoters - GFP.

The Bacillus Calmette-Guerín (BCG) is the only licensed vaccine against tuberculosis. It is an attenuated strain of Mycobacterium bovis obtained after successive passages in glycerol-potato medium for about 15 years, from 1906 to 1920 . $^{(1)}$ Since its introduction in 1921, it has been administered to more than three billion individuals with few reported side effects, being one of the safest vaccines in the world. ${ }^{(1)}$ In addition, features such as low production cost, heat stability, induction of long-lasting type 1 helper T cell (Th1) immunity and potent immunostimulation, have motivated its investigation as a live delivery vector for the development of new vaccines against important infectious diseases..$^{(2,3,4,5,6,7,8)} \mathrm{BCG}$ is also the most successful immunotherapeutic agent for treating nonmuscle invasive bladder cancer. ${ }^{(9)}$ In addition, there are studies implicating BCG vaccination as responsible for non-specific protection against other infectious diseases, also called trained immunity or off-target effects. ${ }^{(10)}$

Pioneering research in the genetic manipulation of Mycobacterium, such as performed by Jacobs Jr et al. allowed the introduction of foreign DNA into Mycobac-

doi: 10.1590/0074-02760190347

Financial support: CNPq (Grant number: 130346/2013-6), FAPESP (Grants numbers: 2014/01271-0 and 2017/24832-6), Fundação Butantan. LVN is a CNPq fellowship; CCS and LCCL are researchers.

+Corresponding author: ivanpnbutantan@gmail.com

(D) orcid.org/0000-0001-9843-1594

Received 13 September 2019

Accepted 13 April 2020 terium smegmatis and BCG. ${ }^{(11)}$ This opened the possibility for development of multi-vaccines based on BCG as a live vehicle to present heterologous antigens from different pathogens. Since then, several groups have had success in the development of rBCG strains, expressing antigens from virus, bacteria or parasites, or even cytokine molecules. ${ }^{(1,2,12)}$ Despite several reports on the successful construction of rBCG strains, many rBCG constructs did not attain expression, revealing the importance of characterising other expression vectors construct. On the other hand, there are studies that have indicated a role for antigen expression level in the induction of immune responses. For the Mycobacterium tuberculosis (Mtb) antigen, 85B, low-level expression skewed the immune response towards a T helper 1 (Th1) immune response, and higher expression levels towards a Th2 immune response. ${ }^{(13)}$

Different features can influence antigen expression in $\mathrm{BCG}$, such as promoter strength, codon usage, vector stability, as well as the strain of BCG. Although, all these characteristics together can influence gene expression, the promoter is surely the key factor of this system. ${ }^{(14,15)}$

Several promoters have been used to express a variety of genes in mycobacteria. For some time, the heat shock promoter, $\mathrm{P}_{\mathrm{Hsp} 60}$, was the preferential choice, in part for being considered a strong promoter. ${ }^{(15)}$ However, several other genes have not been expressed yet, even using strong promoters such as $\mathrm{P}_{\mathrm{Hsp60}}$. The use of other promoters was described, such as the mutated beta-lactamase promoter, $\mathrm{P}_{\mathrm{BlaF}^{*}}$ (from $M$. fortuitum), ${ }^{(12)}$ the alpha antigen promoter, $\mathrm{P}_{\mathrm{Ag}}$ (from $\mathrm{BCG}$ ), the $19 \mathrm{kDa}$ antigen promoter (from $M t b$ ) or the $M$. paratuberculosis $\mathrm{P}_{\mathrm{AN}}$ 
promoter (Table), being $\mathrm{P}_{\mathrm{BlaF}}$ considered the stronger and $\mathrm{P}_{\mathrm{AN}}$ the weaker when compared between them. As consequence, this allowed gene expression at different levels in mycobacteria. The strength of different promoters has been evaluated ${ }^{(15,16)}$ with different reporter genes and plasmids as backbone, making it difficult to compare the studies.

Another feature that is important for antigen expression is vector stability. We have constructed several rBCG strains using the pLA71 plasmid ${ }^{(12,17)}$ as a backbone vector, expressing a genetically detoxified subunit 1, S1PT, ${ }^{(6)} \mathrm{CRM} 197^{(18)}$ and tetanus toxin fragment C. ${ }^{(19)}$ Especially for the rBCG-S1PT construct, we have observed high stability in vivo. ${ }^{(6)}$ On the other hand, it has been shown that maintaining the plasmid based on antibiotic resistance does not determine that the antigen will be expressed over time. ${ }^{(20)}$ Rizze et al. ${ }^{(20)}$ developed an auxotrophic mycobacterial system based on leuD complementation to express $85 \mathrm{~B}$ and $85 \mathrm{BT}$ antigens (BCG $\Delta l e u D-85 \mathrm{~B}$ and $\mathrm{BCG} 4 l e u D-85 \mathrm{BT}$ ), which proved to be stable both for the presence of the plasmid and for the expression of these antigens over a long period of time after immunisation of mice. We have previously obtained a BCG strain auxotrophic for lysine, (BCG$\triangle$-lys $A$ ), complemented with a pLA71-based expression vector $^{(7)}$ which was shown to maintain expression of the antigen through several in vitro passages without antibiotic selection (results not shown). These results indicate the stability of the pLA71-based backbone vectors.

Therefore, considering that it is still important to optimise expression systems for the development of new rBCG strains, ${ }^{(15)}$ the aim of the current work was to obtain a collection of mycobacterial vectors based on a vector backbone previously tested for stability, using a range of promoters with different strengths. The activity of the different promoters was evaluated using the green fluorescent protein (GFP) as reporter for gene expression. In vitro and in vivo stability was also evaluated.

\section{MATERIALS AND METHODS}

Strains - Escherichia coli DH5a, M. smegmatis $\mathrm{mc}^{2}$ 155 and M. bovis BCG strain Moreauwere used in this study. E. coli was grown in Luria-Bertani medium at $37^{\circ} \mathrm{C}$. The $M$. smegmatis derived strains were grown in Middlebrook 7H9 (Difco Laboratories, Detroit, MI) or Middlebrook $7 \mathrm{H} 10$ agar (Difco Laboratories) with $0.5 \%$ glycerol and $0.05 \%$ Tween 80 (MB7H9 and MB7H10, respectively), plus kanamycin $(20 \mu \mathrm{g} / \mathrm{mL})$ when required, at $37^{\circ} \mathrm{C}$ in a humidified $5 \% \mathrm{CO}_{2}$ incubator.

$\mathrm{BCG}$ or the constructs, were grown in $\mathrm{MB} 7 \mathrm{H} 9$ or MB7H10 agar with $10 \%$ OADC, $0.5 \%$ glycerol and $0.05 \%$ Tween 80 , plus kanamycin $(20 \mu \mathrm{g} / \mathrm{mL})$ when required, at $37^{\circ} \mathrm{C}$ in a humidified $5 \% \mathrm{CO}_{2}$ incubator, until cultures reached an optical density (OD) of $\sim 0.8$. Bacteria were harvested by centrifugation at $2.800 \mathrm{xg}$, washed twice with distilled water and resuspended in $10 \%$ Glycerol. The mycobacterial preparations were maintained at $-80^{\circ} \mathrm{C}$ until used and Colony-forming units (CFU) were determined after $48 \mathrm{~h}$.

Construction of the vectors - The $g f p$ gene was amplified from the pEGFP-N1 vector (Clontech Laboratories), including a Kpn I restriction site at the 5' end and a Not I restriction site at the 3' end [Supplementary data (Table)]. The promoters $\mathrm{P}_{\mathrm{Hsp} 60}$ and $\mathrm{P}_{\alpha \mathrm{Ag}}$ were amplified by polymerase chain reaction (PCR) using genomic DNA isolated from $\mathrm{BCG}$ as template. The $\mathrm{P}_{\mathrm{BlaF}}, \mathrm{P}_{\mathrm{AN}}$ and $\mathrm{P}_{\mathrm{LS}}$ promoters were amplified by PCR from plasmids containing the sequences, previously used in our lab. ${ }^{(5,6,8,14)}$

A BamH I restriction site was included at the 5' end and a Kpn I restriction site was included at the 3' end of the promoters [Supplementary data (Table)]. These fragments, promoters and $g f p$, were fused by Doublejoint PCR technique, according to $\mathrm{Yu}$ et al. ${ }^{(21)}$ The expression cassettes were formed and cloned into the pLA71 vector at the BamH I and Not I sites. DH5a E. coli transformed with the expression vectors resulted in colonies that were screened by colony PCR for the presence of the respective fusion gene inserts. DNA from

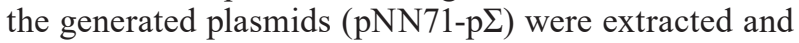
the correct constructs were confirmed by sequencing. $\mathrm{pJH} 223-\mathrm{P}_{\mathrm{L} 5}-g f p$, is derived from an integrative vector, pJH223, and kindly provided by Dr William Jacobs (Albert Einstein College of Medicine - NY, USA).

Transformation of mycobacteria - Aliquots $(100 \mu \mathrm{L})$ of competent mycobacterial cells were electroporated with 1-2 $\mu \mathrm{g}$ of DNA with a single pulse $(2.5 \mathrm{kV} ; 25 \mu \mathrm{F}$; 600 ohms) using a Bio-Rad Gene Pulser. Transformed M. smegmatis cells were incubated in $4 \mathrm{~mL}$ MB7H9 medium at $37^{\circ} \mathrm{C}$ for $3 \mathrm{~h}$; transformed $\mathrm{BCG}$ was incubated under the same conditions for $24 \mathrm{~h}$, before plating in MB7H10 containing kanamycin $(20 \mu \mathrm{g} / \mathrm{mL})$. Plates were

TABLE

General features of the promoter used in this study

\begin{tabular}{lccc}
\hline Promoter & Strength & Source & Reference \\
\hline $\mathrm{P}_{\mathrm{AN}}$ & Low & Mycobacterium paratuberculosis & Murray et al. $^{(30)}$ \\
\hline $\mathrm{P}_{\mathrm{Hsp60}}$ & High & Bacillus Calmette-Guérin (BCG) & Stover et al. $^{(2)}$ \\
\hline $\mathrm{P}_{\alpha \mathrm{Ag}}$ & Moderate & M. kansassi & Matsuo et al. $^{(31)}$ \\
$\mathrm{P}_{\mathrm{BlaF} *}$ & High & M. fortuitum & Timm et al. $^{(12)}$ \\
\hline $\mathrm{P}_{\text {Left }}$ & $\mathrm{ND}$ & Micobacteriophage L5 & Nesbit et al. $^{(32)}$ \\
\hline
\end{tabular}

ND: not determined until now. 
incubated at $37^{\circ} \mathrm{C}$ in a humidified $5 \% \mathrm{CO}_{2}$ incubator: $M$. smegmatis for three days and three weeks for BCG. Individual clones were cultivated in MB7H9, washed and stored frozen in phosphate buffered saline (PBS) and 10\% glycerol at $-80^{\circ} \mathrm{C}$ until used. Individual mycobacterial colonies were analysed directly using a Nikon Eclipse E200 fluorescence microscope. Transformation of BCG with these vectors generated the following constructs: rBCGpLA71- $\mathrm{P}_{\alpha \mathrm{g}}-g f p, \quad$ rBCG-pLA71-P $\mathrm{P}_{\mathrm{AN}}-g f p, \quad$ rBCG-pLA71$\mathrm{P}_{\mathrm{Hsp} 60}-\mathrm{g} f$ pand BCG-pJH223- $\mathrm{P}_{L 5}-\mathrm{gfp}$.

Flow cytometric analysis of fluorescent bacteria - To measure the fluorescence in the mycobacterial transformants expressing GFP, samples were thawed and resuspended in PBS. The geometric mean fluorescence was determined for the bacterial populations by flow cytometry using a FACSCanto II (BD Biosciences).

Infection of a macrophage cell line by the $r B C G$ pNN71-p Sstrains and confocal microscopy analysis The RAW 264.7 macrophage cell line was gently provided by Dr Maria Cristina Breno, from the Laboratory of Pharmacology, Institute Butantan. These cells were grown in Roswell Park Memorial Institute (RPMI) (Gibco-BRL) supplemented with $10 \%$ heat-inactivated foetal calf serum (FCS) (Gibco-BRL) at $37^{\circ} \mathrm{C}$ in a humidified $5 \% \mathrm{CO}_{2}$ incubator. The cells were seeded in 24-well plates (Corning) $\left(1 \times 10^{6}\right.$ cells per well) and allowed to adhere overnight. The macrophages were then incubated with the mycobacterial suspensions at a multiplicity of infection (MOI) 1:10. After $3 \mathrm{~h}$ of infection, macrophages were washed to remove the remaining extracellular bacteria and the cells were incubated for an additional $24 \mathrm{~h}$ in fresh medium. The preparation of samples for confocal analysis was made as follow: cells were adhered to glass slides coated with poly-${ }_{\mathrm{L}}$-lysine (Sigma-Aldrich), fixed in $4 \%$ paraformaldehyde and quenched with $0.1 \mathrm{M}$ glycine. Mounted slides were analysed with a confocal laser microscope system (Zeiss LSM 510).

Immunisation of mice with $r B C G$-GFP strains and recovery of mycobacteria from the spleens - Five female $\mathrm{BALB} / \mathrm{c}$ mice, five to seven weeks, were inoculated i.p. with the rBCG-pLA71- $\mathrm{P}_{\mathrm{AN}^{\mathrm{N}}}-g f p$ or rBCG-pLA71- $\mathrm{P}_{\mathrm{Hsp} 60^{-}}$ $g f p$ strains at a concentration of $1 \times 10^{6} \mathrm{CFU} /$ animal. After 30 days, the spleens were extracted, homogenised and serially diluted to be plated with (+) or without (-) kanamycin (Kan) for determination of colony forming units (CFU). Expression of GFP in the colonies were confirmed by fluorescence microscopy in all clones obtained. The bars show the mean plus the standard deviation of five animals per experiment.

Statistical analysis - Statistical analysis between groups were performed using Turkey test. The level of statistical significance was set at $\mathrm{p}<0.001$.

Ethics - All animal experiments were performed according to Brazilian and international guidelines on animal experimentation and approved by the Ethics Committee of Instituto Butantan, São Paulo-SP (CEUAIB), (Permit number 1264/14).

\section{RESULTS}

Construction of mycobacterial expression vectors containing promoters with different strengths using the pLA71 vector system - To compare the strength of different promoters in mycobacteria, in a same vector backbone, we cloned known promoter sequences (Table) into the pLA71 vector system, placing the $g f p$ gene under the control of each promoter (Fig. 1). An expression vector containing the recently characterised promoter from mycobacteriophage L5 $\left(\mathrm{P}_{\mathrm{L} 5}\right),{ }^{(14)}$ was also constructed using
A

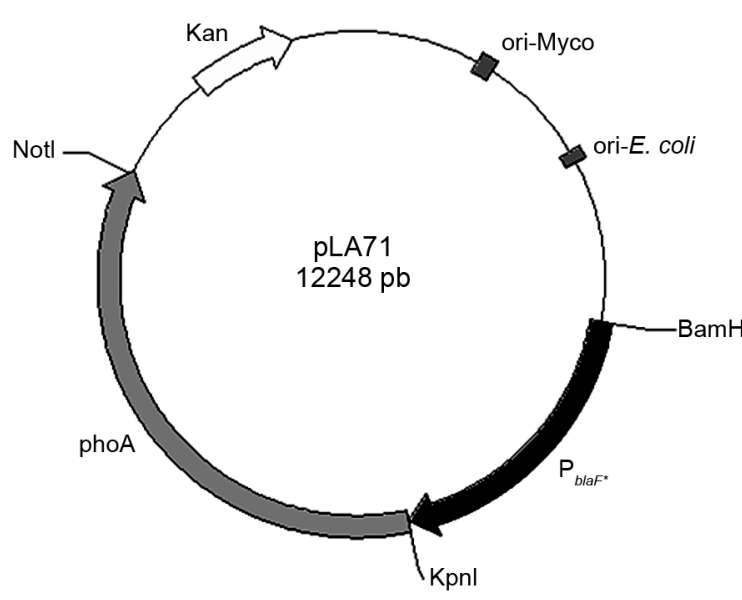

B
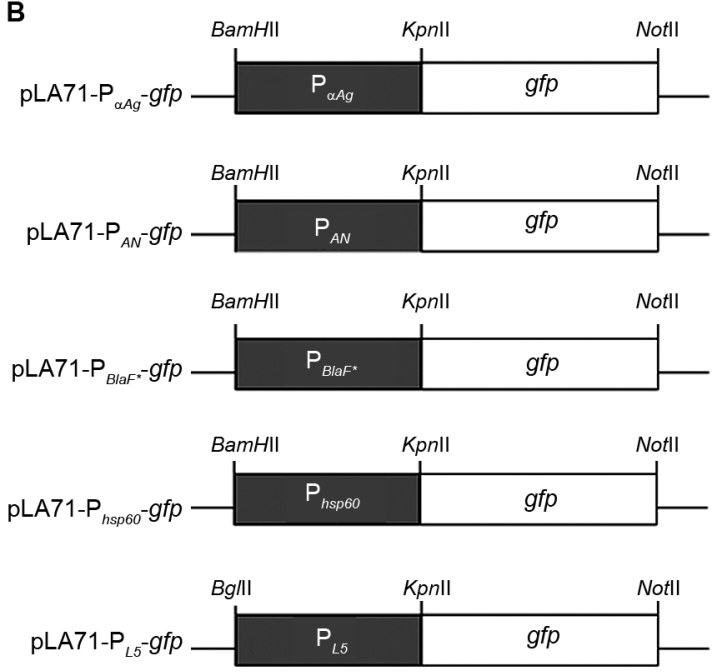

Fig. 1: schematic representation of the pLA71 vector and the expression cassettes formed by the promoters and the $g f p$ reporter gene. (A) pLA71 contains the $\beta$-lactamase promoter $\left(\mathrm{P}_{\mathrm{BlaF}}\right)$, the kanamycin resistance gene $(\mathrm{Kan})$, the Escherichia coli origin of replication (ori-E. coli) and mycobacterial origin of replication (ori-Myco), and the alkaline phosphatase gene (phoA). (B) The expression cassettes constructed using the mycobacterial promoters and the $g f p$ gene by polymerase chain reaction (PCR). 
the same strategy. The different constructs were used to transform M. smegmatis and BCG and the transformants were selected initially by kanamycin resistance and then by GFP expression observed in a fluorescence microscope. Only colonies that showed fluorescence in the plates were expanded in liquid medium to be analysed by flow cytometry. It was possible to obtain clones for all constructs in M. smegmatis and BCG, except for the pLA71- $\mathrm{P}_{\mathrm{L} 5}-g f p$ vector. Interestingly, the $E$. coli colonies transformed with the pLA71- $\mathrm{P}_{L 5}-g f p$ vector displayed a green phenotype, indicating that the construct was functional. Although toxicity of GFP has not been previously described, ${ }^{(14,22,23,24)}$ it is possible that a higher expression of GFP would be toxic to the mycobacteria.

Differential fluorescence of the recombinant $M$. smegmatis strains containing the different expression vector systems - The expression of GFP by the different constructs in M. smegmatis was evaluated by flow cytometry. Two or more colonies from each recombinant M. smegmatis (rSmeg) transformants were expanded in liquid medium and washed in PBS before analysis by flow cytometry. It was possible to observe that the different expression systems presented a variety of fluorescence intensities (Fig. 2). The expression vector based on the $\mathrm{P}_{\mathrm{aA}}$ promoter displayed very low fluorescence in the pLA71 background, comparable to wtBCG. The $\mathrm{P}_{\mathrm{AN}}$ and the $\mathrm{P}_{\mathrm{Hspb} 60}$ promoters displayed intermediate fluorescence intensity and the $\mathrm{P}_{\text {BlaF* }}$ promoter was shown to be the strongest.

Differential fluorescence of the recombinant $B C G$ strains containing the different expression vector systems - Analysing the same vectors in the BCG host, the expression based on the $\mathrm{P}_{\mathrm{aAg}}$ promoter again displayed no significant fluorescence when compared to the wtBCG control. However, in this host, the $\mathrm{P}_{\mathrm{AN}}$ and the $\mathrm{P}_{\text {BlaF* }}$ promoters displayed intermediate fluorescence intensities and the $\mathrm{P}_{\mathrm{Hsp} 60}$ promoter was shown to be the stron- gest (Fig. 3). The range of fluorescence obtained in the rBCG strains was comparable to that obtained inrSmeg strains (Figs 2-3). On the other hand, as was not possible

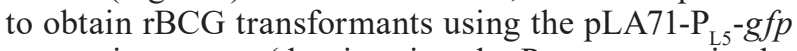
expression vector (that is using the $\mathrm{P}_{\mathrm{L} 5}$ promoter in the pLA71 backbone), we decided to use the integrative vector $\mathrm{pJH} 223-\mathrm{P}_{L 5}-g f p$ containing the $\mathrm{P}_{\mathrm{L} 5}$ promoter previously characterised to verify and compare the expression level of GFP in $\mathrm{rBCG}$ with the pLA71- $\mathrm{P}_{\mathrm{Hsp} 60}-g f p$ vector (Fig. 4).

Analyses of GFP expression in macrophages - To investigate the activity of each promoter in vitro, the macrophage RAW 264.7 cell line was infected with the different rBCG constructs and analysed by confocal microscopy. GFP fluorescence can be observed from inside the macrophages with all rBCG strains (Fig. 5). The rBCG-pLA71- $\mathrm{P}_{\mathrm{AN}}-g f p$ displayed lower fluorescence then rBCG-pLA71-P $\mathrm{B}_{\text {BlaF* }}-g f p$ and rBCG-pLA71- $\mathrm{P}_{\mathrm{Hsp} 60}-g f p$, and the difference between the latter two was not so evident.

Shuttle vectors stability in vivo - In order to determine the in vivo vector stability of the rBCG-GFP constructs, the strains displaying the lowest and the highest expression levels were used, rBCG-pLA71- $\mathrm{P}_{\mathrm{AN}}-g f p$ and rBCG-pLA71- $\mathrm{P}_{\mathrm{Hsp} 60}-g f p$, respectively. BALC/c mice were inoculated i.p. with the rBCG strains and the mycobacteria were recovered from the spleens 30 days later and plated in the presence and absence of kanamycin. It can be observed that there is no difference between the number of bacteria recovered from the two strains, indicating that they have comparable stability (Fig. 6). Furthermore, the presence of kanamycin did not decrease the proportion of bacteria, indicating that the vector was relatively stable in vivo, that is, in the absence of antibiotic pressure. Furthermore, it could be observed that all the colonies recovered from the plates containing kanamycin displayed fluorescence.
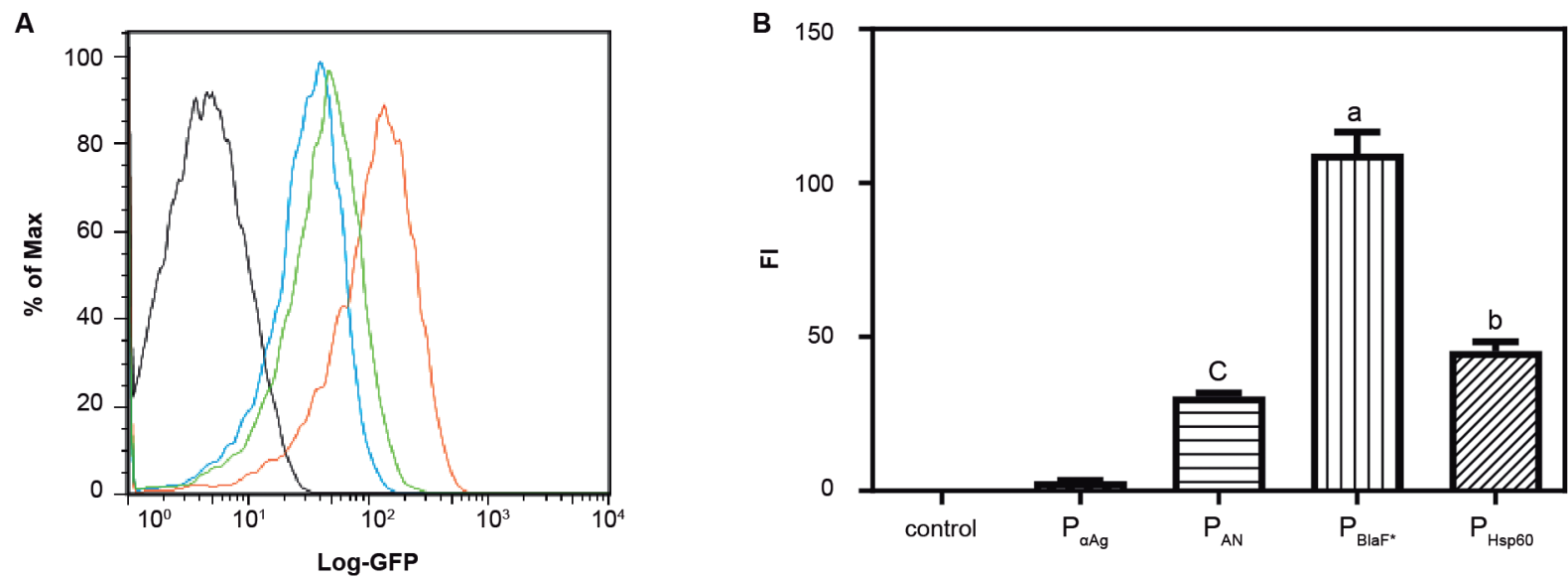

Fig. 2: expression of $g f p$ in Mycobacterium smegmatis transformed with the pNN71-p $\Sigma$ plasmids. (A) Fluorescence histogram obtained from the rSmeg strains containing the pLA71 vectors with the different promoters: Grey, wtSmeg control (not visible); black, $\mathrm{pLA} 71-\mathrm{P}_{\alpha \mathrm{Ag}}{ }^{-g} f p$; blue,

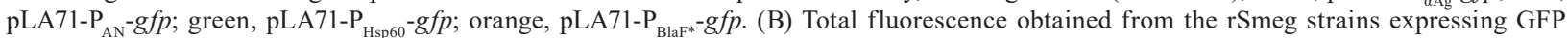
through the different promoters. Results are mean \pm standard deviation (SD). of five colonies from each construct in rSmeg. Differences were considered statistically significant when $\mathrm{p} \leq 0.001$ : (a) as compared to all groups; (b) as compared to the $\mathrm{P}_{\mathrm{AN}}$ group and (c) as compared to the wtSmeg control and $\mathrm{P}_{\alpha \mathrm{Ag}}$ (one-way ANOVA). 

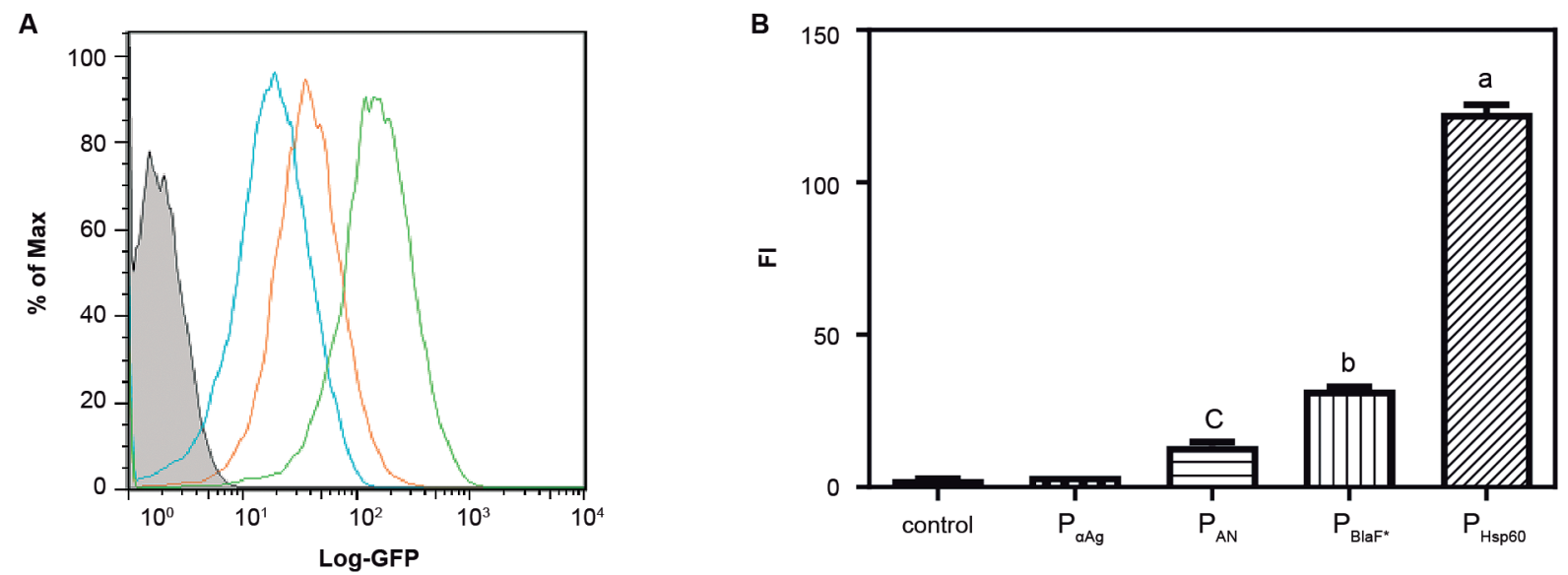

Fig. 3: expression of $g f p$ in Mycobacterium bovis Bacillus Calmette-Guérin (BCG) transformed with the pNN71-p $\Sigma$ plasmids. (A) Fluorescence histogram obtained from the rBCG strains containing the $\mathrm{pLA71}$ vectors with the different promoters: grey, wtBCG control; black, $\mathrm{pLA71-P}_{\alpha \mathrm{Ag}}{ }^{-}$

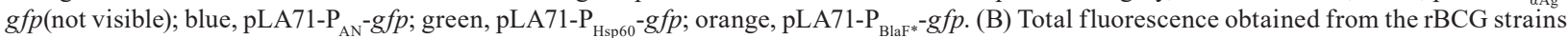
expressing GFP through the different promoters. Results are mean \pm standard deviation (SD) of five colonies from each construct in rBCG. Differences were considered statistically significant when $\mathrm{p} \leq 0.001$ : (a) as compared to all groups; (b) as compared to the $\mathrm{P}_{\mathrm{AN}}$ group; and(c) as compared to the wtBCG control and $\mathrm{P}_{\alpha \mathrm{Ag}}$ (one-way ANOVA).

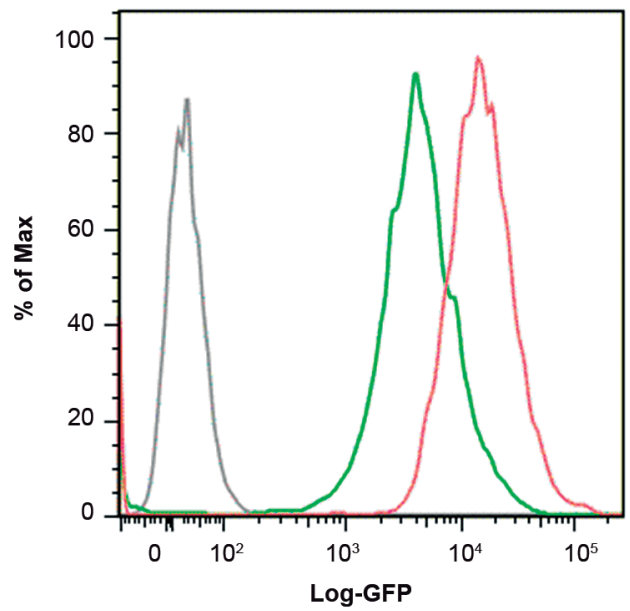

Fig. 4: expression of green fluorescence protein (GFP) in Bacillus Calmette-Guérin (BCG) transformed with the pLA71- $\mathrm{P}_{\mathrm{Hsp} 60}-g f p$ plasmid or the $\mathrm{pJH} 223-\mathrm{P}_{\mathrm{L}}-\mathrm{g} f \mathrm{p}$ integrative vector. Histogram of fluorescence obtained from rBCG transformed with the vectors. The expression levels in $\mathrm{rBCG}$ were detected using flow cytometry. Grey, wtBCG control; green, rBCG-pLA71- $\mathrm{P}_{\mathrm{Hsp} 60}-g f p$; and red, rBCGpJH223-P ${ }_{\text {L5 }}-g f p$.

\section{DISCUSSION}

BCG has been used as a vaccine against tuberculosis for almost a century, being the most widely used vaccine in the world.(15) It is a potent modulator of the immune response, which makes it a very attractive live vehicle for the presentation of heterologous antigens. However, important issues such as vector stability and antigen expression levels have been shown to play major roles in the success of different constructs of recombinant BCG vaccines. Most of the replicative vectors for mycobacteria were obtained using the pLA5000 origin of replication, which allows three to ten plasmid copies per bacterial cell. ${ }^{(12,25)}$ On the other hand, the level of expression of heterologous genes in mycobacteria is modulated mainly by the promoter, yet not solely. Although, there are several studies performed with a set of promoters and in different vectors as backbone, ${ }^{(20,26)}$ investigations in the search for new and improved promoters continue to be performed. ${ }^{(25,27)}$

We have extensive experience with the mycobacterial expression vector pLA71, containing the $\mathrm{P}_{\mathrm{BlaF}}$ promoter, which we have used to express many different antigens from different pathogens. Our experience has shown that this vector backbone is considerably stable in vitro and in vivo, including when used in an auxotrophic complementation strategy. ${ }^{(7)}$ Therefore, this vector backbone would be convenient to be used in future constructs. On the other hand, vaccines for different pathogens can require different antigen expression levels to achieve induction of appropriate immune responses. Therefore, it is important to compare promoters with different strengths in a same backbone shown to be stable.

In the present study, we have constructed a set of vectors based on the pLA71 backbone, containing a selection of promoters covering a range of expression strengths. In addition to these traditional promoters, we also evaluated a mycobacteriophage promoter, $\mathrm{P}_{\mathrm{L} 5}$ recently studied in our laboratory, considered strong, but never compared with others in the same study. ${ }^{(14)}$

We investigated the expression levels of the promoters considered to be weak, moderate and strong (Table) in the pLA71 background and in two species of mycobacteria, M. smegmatis and BCG. Our results showed that the $\mathrm{P}_{\mathrm{AN}}$ promoter, which is considered the weakest among those evaluated, was also the weakest in both species, indicating that this promoter shows consistent results in different backgrounds and across species. However, the $\mathrm{P}_{\mathrm{aAg}}$ promoter, considered to be moderate, did not present significant fluorescence in either species when compared to BCG control. Furthermore, it was also not possible to visualise green colonies under the fluorescence microscope (data not shown). It is possible that this promoter suffers some negative influence in the pLA71 background. 
Furthermore, between the promoters considered to be strong $\left(\mathrm{P}_{\mathrm{BlaF}}\right.$ and $\left.\mathrm{P}_{\mathrm{Hsp} 60}\right)$, the difference between them was shown to be dependent on the species, since $\mathrm{P}_{\mathrm{BlaF}}$ was shown to be stronger in M. smegmatis and $\mathrm{P}_{\mathrm{Hsp} 60}$ stronger in $\mathrm{BCG}$ (as observed in vitro in liquid medium). However, when we tested recombinant BCG strains in a macrophage infection assay and visualised them by confocal microscopy, strains driving expression of GFP by $\mathrm{P}_{\text {BlaF* }^{*}}$ and $\mathrm{P}_{\mathrm{Hsp} 60}$ seem not to be different, suggesting that the regulatory mechanisms for these two promoters can be influenced by the environment.

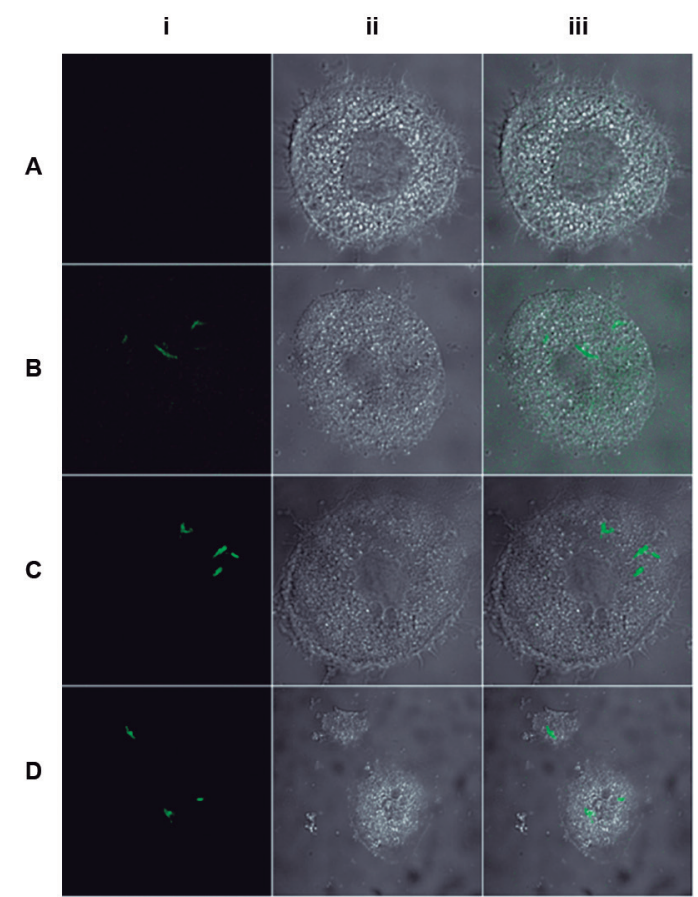

Fig. 5: expression of the different rBacillus Calmette-Guérin-green fluorescence protein (rBCG-GFP) strains in the macrophage cell line, RAW 264.7. Confocal microscopy analysis of GFP expression in rBCG-pLA71- $\mathrm{P}_{\mathrm{AN}}-g f p(\mathrm{~B}), \mathrm{rBCG}-\mathrm{pLA71- \textrm {P } _ { \mathrm { BlaF } ^ { * } }}-g f p(\mathrm{C})$ and rBCGpLA71- $\mathrm{P}_{\mathrm{Hsp} 60}-g f p$ (D) inside the macrophage cell line, RAW 264.7. wtBCG was used as negative control (A). (i) FITC, (ii) Visible and (iii) FITC plus visible.
When analysing the same promoter in both $\mathrm{Myco}$ bacterium species we can observe that $\mathrm{P}_{\text {BlaF* }}$ induced fluorescence activity about 3-fold higher in M. smegmatis than in BCG. On the contrary, for $\mathrm{P}_{\mathrm{Hsp} 66}$, the fluorescence activity was about 3-fold higher in BCG than in $M$. smegmatis. In a similar study, using the $\mathrm{P}_{\mathrm{Hsp} 60}$ promoter and $\beta$-galactosidase activity as reporter in M. smegmatis and BCG under the same culture conditions, Dellagostin et al. observed a higher expression level in M. smegmatis. ${ }^{(26)}$ This contrasts with our results, but it is important to consider that these experiments used different reporter proteins, suggesting the importance of the gene sequence in the expression levels obtained.

$\mathrm{P}_{\mathrm{L} 5}$ is considered a strong promoter, however, it has not been compared to other promoters in a common expression vector for mycobacteria. We have previously investigated the original promoter $\mathrm{P}_{\mathrm{L} 5}$ cloned into the pJH152 vector for expression of GFP and submitted to randomised mutation through error-prone PCR. The obtained $\mathrm{P}_{\mathrm{L} 5}$ mutant promoters were compared seeking the higher or lower GFP expression levels in BCG. (14) We could not compare our construct pLA71-pL5-gfp to the pJH152-pL5-gfp because our construct does not generate $M$. smegmatis or BCG transformants, although its functionality was demonstrated by high expression of GFP in E. coli clones. Perhaps, the expression level obtained by Kanno et al. ${ }^{(14)}$ is not high enough to be toxic, unlike ours. Furthermore, in a recent work, Oliveira et al. ${ }^{(28)}$ have compared several different promoters using the pUP500 vector as a backbone and the eGFP as reporter protein. However, although this work has the same idea, the purpose for Oliveira et al. ${ }^{(28)}$ was to employ the Biobrick strategy to construct a toolbox of several mycobacterial vector parts, including promoters and reporter genes. In fact, similar to our work, it is an effort to standardise a system that can enhance the efficacy and use of recombinant BCG.

Jain et al. ${ }^{(29)}$ measured the GFP expression by mycobacteriophages within mycobacteria using $\mathrm{P}_{L 5}$ phage promoter in comparison with that obtained by the $\mathrm{P}_{\mathrm{Hsp} 60}$ promoter in the same system. They showed that the fluorescence activity was almost 100 -fold higher in the first. Interestingly, transformants of $\mathrm{pLA} 71-\mathrm{P}_{\mathrm{L} 5}-\mathrm{g} f \mathrm{p}$ could not
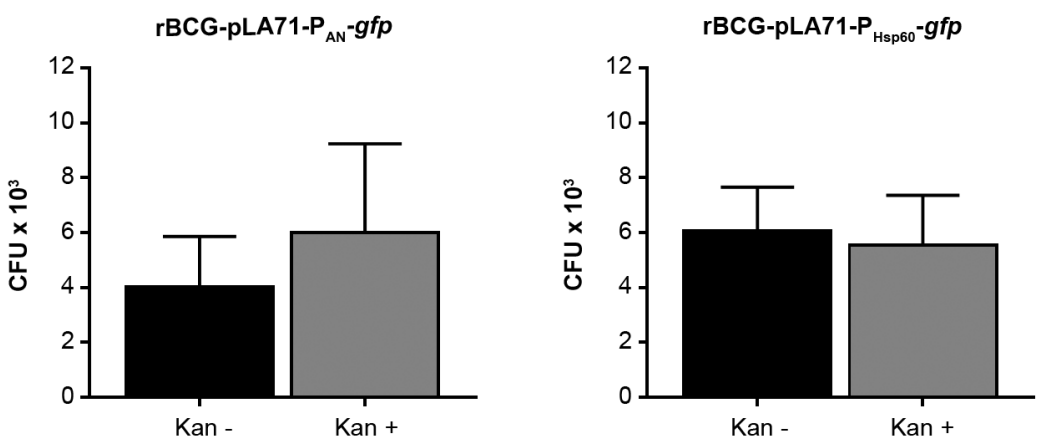

Fig. 6: comparable stability of the vectors expressing high and low levels of green fluorescence protein (GFP) in vivo. Five female BALB/c mice, five to seven weeks, were inoculated i.p. with the rBCG-pLA71- $\mathrm{P}_{\mathrm{AN}}-g f p$ and rBCG-pLA71- $\mathrm{P}_{\mathrm{Hsp} 60}-g f p$ strains at a concentration of $1 \times 10^{6}$ colony forming units (CFU)/animal. After 30 days, the spleens were extracted, homogenised and serially diluted to be plated with $(+)$ or without $(-)$ kanamycin (Kan) for determination of CFU. The bars show the mean plus the standard deviation of five animals per experiment. 
be selected in either mycobacterial species, although the plasmid was functional in E. coli since we could observe green colonies (data not shown). It is possible that this can be due to the combination of the high strength of the promoter and the plasmid copy number (up to ten copies). The higher expression level may produce toxic amounts of the protein for the mycobacteria. When we used the integrative plasmid, $\mathrm{pJH} 223-g f p$ (which has only one copy), fluorescence activity was higher than with $\mathrm{P}_{\mathrm{Hsp60}}$, showing the strongest expression level within the $\mathrm{rBCG}$ strains studied (Fig. 4). Integrative vectors are known to allow greater stability but lower expression levels. Once transformants of pLA71- $\mathrm{P}_{\mathrm{L} 5}-g f p$ were not obtained, it was not possible to compare the stability between episomal and integrative vectors in this study. However, greater levels of GFP expression were observed using pJH223- $\mathrm{P}_{L 5}-g f p$ vector, and even with only one copy, it shows higher expression levels that an episomal vector containing $\mathrm{P}_{\mathrm{Hsp} 60}$ for instance.

We have obtained a series of vectors to be used in the development of $\mathrm{rBCG}$ strains with previously determined level of antigen expression and vector stability, that can lead to more successful outcomes in $\mathrm{rBCG}$ vaccines.

\section{ACKNOWLEDGEMENTS}

To Arleíde Carvalho and Marlene F Silva for technical assistance.

\section{AUTHORS' CONTRIBUTION}

LCCL - Coordinated the study; LCCL and IPN - conceived and designed the experiments; LVN, CCS and IPN - performed the experiments; LCCL and IPN - wrote the paper and revised it critically. All authors contributed to the interpretation of the results and have read and approved the final manuscript.

\section{REFERENCES}

1. Bastos RG, Borsuk S, Seixas FK, Dellagostin OA. Recombinant Mycobacterium bovis BCG. Vaccine. 2009; 27(47): 6495-503.

2. Stover CK, de la Cruz VF, Fuerst TR, Burlein JE, Benson LA, Bennett LT, et al. New use of BCG for recombinant vaccines. Nature. 1991; 351(6326): 456-60.

3. Andrade PM, Chade DC, Borra RC, Nascimento IP, Villanova FE, Leite LCC, et al. The therapeutic potential of recombinant BCG expressing the antigen S1PT in the intravesical treatment of bladder cancer. Urol Oncol-Semin Ori. 2010; 28(5): 520-5.

4. Chade DC, Borra RC, Nascimento IP, Villanova FE, Leite LCC, Andrade E, et al. Immunomodulatory effects of recombinant BCG expressing pertussis toxin on TNF-alpha and IL-10 in a bladder cancer model. J Exp Clin Canc Res. 2008; 27: 78.

5. Kanno AI, Goulart C, Leite LCC, Pagliarone AC, Nascimento IP. A bivalent recombinant Mycobacterium bovis BCG expressing the S1 subunit of the pertussis toxin induces a polyfunctional CD4(+) T cell immune response. BioMed Res Int. 2019; 2019: 9630793.

6. Nascimento IP, Dias WO, Mazzantini PP, Miyaji EN, Gamberini $\mathrm{M}$, Quintilio W, et al. Recombinant Mycobacterium bovis BCG expressing pertussis toxin subunit $\mathrm{S} 1$ induces protection against an intracerebral challenge with live Bordetella pertussis in mice. Infect Immun. 2000; 68(9): 4877-83.

7. Nascimento IP, Dias WO, Quintilio W, Hsu T, Jacobs WR, Leite LCC. Construction of an unmarked recombinant BCG expressing a pertussis antigen by auxotrophic complementation: Protection against Bordetella pertussis challenge in neonates. Vaccine. 2009; 27(52): 7346-51.
8. Nascimento IP, Rodriguez D, Santos CC, Amaral EP, Rofatto $\mathrm{HK}$, Junqueira-Kipnis AP, et al. Recombinant BCG expressing LTAK63 adjuvant induces superior protection against Mycobacterium tuberculosis. Sci Rep. 2017; 7(1): 2109.

9. Donin NM, Lenis AT, Holden S, Drakaki A, Pantuck A, Belldegrun A, et al. Immunotherapy for the treatment of urothelial carcinoma. J Urol. 2017; 197(1): 14-22.

10. Uthayakumar D, Paris S, Chapat L, Freyburger L, Poulet H, De Luca K. Non-specific effects of vaccines illustrated through the BCG example: from observations to demonstrations. Front Immunol. 2018; 9: 2869.

11. Jacobs WR, Tuckman M, Bloom BR. Introduction of foreign DNA into Mycobacteria using a shuttle phasmid. Nature. 1987; 327(6122): 532-5.

12. Timm J, Perilli MG, Duez C, Trias J, Orefici G, Fattorini L, et al. Transcription and expression analysis, using lac $Z$ and $p h o A$ gene fusions, of Mycobacterium fortuitum $\beta$-lactamase genes cloned from a natural isolate and a high-level $\beta$-lactamase producer. Mol Microbiol. 1994; 12(3): 491-504.

13. Dhar N, Rao V, Tyagi AK. Skewing of the Th1/Th2 responses in mice due to variation in the level of expression of an antigen in a recombinant BCG system. Immunol Lett. 2003; 88(3): 175-84.

14. Kanno AI, Goulart C, Rofatto HK, Oliveira SC, Leite LCC, McFadden J. New recombinant Mycobacterium bovis BCG expression vectors: improving genetic control over mycobacterial promoters. Appl Environ Microbiol. 2016; 82(8): 2240-6.

15. Oliveira TL, Rizzi C, Dellagostin OA. Recombinant BCG vaccines: molecular features and their influence in the expression of foreign genes. Appl Microbiol Biotechnol. 2017; 101(18): 6865-77.

16. Al-Zarouni M, Dale JW. Expression of foreign genes in Mycobacterium bovis $\mathrm{BCG}$ strains using different promoters reveals instability of the $h s p 60$ promoter for expression of foreign genes in Mycobacterium bovis BCG strains. Tuberculosis. 2002; 82(6): 283-91.

17. Lim EM, Rauzier J, Timm J, Torrea G, Murray A, Gicquel B, et al. Identification of Mycobacterium tuberculosis DNA sequences encoding exported proteins by using $p h o A$ gene fusions. J Bacteriol. 1995; 177(1): 59-65.

18. Mazzantini RP, Miyaji EN, Dias WO, Sakauchi D, Nascimento AL, Raw I, et al. Adjuvant activity of Mycobacterium bovis BCG expressing CRM197 on the immune response induced by BCG expressing tetanus toxin fragment C. Vaccine. 2004; 22(5-6): 740-6.

19. Miyaji EN, Mazzantini RP, Dias WO, Nascimento AL, Marcovistz R, Matos DS, et al. Induction of neutralizing antibodies against diphtheria toxin by priming with recombinant Mycobacterium bovis BCG expressing CRM(197), a mutant diphtheria toxin. Infect Immun. 2001; 69(2): 869-74.

20. Rizzi C, Peiter AC, Oliveira TL, Seixas Neto ACP, Leal KS, Hartwig DD, et al. Stable expression of Mycobacterium bovis antigen 85B in auxotrophic M. bovis bacillus Calmette-Guérin. Mem Inst Oswaldo Cruz. 2017; 112(2): 123-30.

21. Yu JH, Hamari Z, Han KH, Seo JA, Reyes-Dominguez Y, Scazzocchio C. Double-joint PCR: a PCR-based molecular tool for gene manipulations in filamentous fungi. Fungal Genet Biol. 2004; 41(11): 973-81.

22. Mutoji KN, Ennis DG. Expression of common fluorescent reporters may modulate virulence for Mycobacterium marinum: dramatic attenuation results from Gfp over-expression. Comp Biochem Physiol C Toxicol Pharmacol. 2012; 155(1): 39-48.

23. Barker LP, Porcella SF, Wyatt RG, Small PL. The Mycobacterium marinum G13 promoter is a strong sigma 70 -like promoter that is expressed in Escherichia coli and mycobacteria species. FEMS Microbiol Lett. 1999; 175(1): 79-85. 
24. Triccas JA, Britton WJ, Gicquel B. Isolation of strong expression signals of Mycobacterium tuberculosis. Microbiology. 2001; 147(Pt 5): 1253-8

25. Lee H, Kim BJ, Kim BR, Kook YH, Kim BJ. The development of a novel Mycobacterium-Escherichia coli shuttle vector system using pMyong2, a linear plasmid from Mycobacterium yongonense DSM 45126T. PLoS One. 2015; 10(3): e0122897.

26. Dellagostin OA, Esposito G, Eales LJ, Dale JW, McFadden J. Activity of mycobacterial promoters during intracellular and extracellular growth. Microbiology. 1995; 141(Pt 8): 1785-92.

27. Eitson JL, Medeiros JJ, Hoover AR, Srivastava S, Roybal KT, Ainsa JA, et al. Mycobacterial shuttle vectors designed for highlevel protein expression in infected macrophages. Appl Environ Microbiol. 2012; 78(19): 6829-37.

28. Oliveira TL, Stedman A, Rizzi C, Dorneles J, da Cunha CEP, Junior ASV, et al. A standardized BioBrick toolbox for the assembly of sequences in mycobacteria. Tuberculosis. 2019; 119: 101851.
29. Jain P, Hartman TE, Eisenberg N, O'Donnell MR, Kriakov J, Govender K, et al. phi(2)GFP10, a high-intensity fluorophage, enables detection and rapid drug susceptibility testing of Mycobacterium tuberculosis directly from sputum samples. J Clin Microbiol. 2012; 50(4): 1362-9.

30. Murray A, Winter N, Lagranderie M, Hill DF, Rauzier J, Timm J, et al. Expression of Escherichia coli? - galactosidase in Mycobacterium bovis BCG using an expression system isolated from Mycobacterium paratuberculosis which induced humoral and cellular immune responses. Mol Microbiol. 1992; 6(22): 3331-42.

31. Matsuo K, Yamaguchi R, Yamazaki A, Tasaka H, Terasaka K, Totsuka M, et al. Establishment of a foreign antigen secretion system in mycobacteria. Infect Immun. 1990; 58(12): 4049-54.

32. Nesbit CE, Levin ME, Donnelly-Wu MK, Hatfull GF. Transcriptional regulation of repressor synthesis in mycobacteriophage L5. Mol Microbiol. 1995; 17(6): 1045-56. 